\title{
Multilayers Plates Buckling by Mixed Finite Elements
}

\author{
Roberto S. Carnicer, Braian A. Desía and Rodolfo A. Schwarz \\ Faculty of Engineering, University of Belgrano, Buenos Aires C1059ABQ, Argentina
}

\begin{abstract}
It is presented a buckling analysis of multilayered plates by the finite elements method with mixed unknowns (displacement and rotations, and transverse interlaminar stresses). In the mixed model, each layer is analyzed as a single plate, where the continuity of displacement is ensured by Lagrange multipliers which represent static variables. This procedure is easy to be implemented from the computational point of view. A methodology to solve the eigen problem is presented based on the inverse iteration method. The model is verified successfully with results obtained by other authors.
\end{abstract}

Key words: Multilayered plates, buckling, finite elements, interlaminar stresses.

\section{Introduction}

Advanced composite materials are widely used in many engineering branches such as civil engineering, naval engineering and aerospace engineering because of their high strength-to-weight ratio, excellent corrosion resistance, good fatigue behavior and other superior properties with respect to conventional materials.

Within the different types of composite materials, laminates are the most popular due to their variety of structural applications in situations where high membrane resistance and flexural strength are required. The composite laminates are basically plates formed by several sheets that are perfectly joined together, presenting an anisotropic behavior. Each sheet is composed of fibers embedded in a matrix. These fibers give high mechanical properties to the sheet in the direction of the fiber, while the matrix holds them together. The sheets are placed one on top of the other oriented according to the design requirements, in order to optimize the use of the material. Their increasing use demands the development of efficient and precise numerical methods to adequately predict their complex behavior.

Corresponding author: Roberto S. Carnicer, professor, engineer; research fields: continuous mechanics, finite elements and multilayer plates.
In the present article, a solution, based on a layered model, is proposed for the determination of the critical buckling load from finite elements with mixed variables (kinematic and static).

\section{Problem Formulation}

Let a plate composed of $n$ layers of height $h_{i}$, perfectly connected and whose mechanical properties may differ, define a particular Cartesian coordinate system $\left\{z^{\alpha}, \alpha=1,2,3\right\}$ for each layer on its respective mean surface $\Omega_{i}$, where $\left(z^{1}, z^{2}\right)$ are the coordinates in the plane, $\left(z^{3}\right)$ is the transverse coordinate. The upper face of each is in $z^{3}=-h_{i} / 2$ and the lower face in, $z^{3}=+h_{i} / 2$.

The kinematics of each layer is modeled independently by adopting the FSDT (first-order shear deformation theory). Thus, the field of displacement of a point $\left\{z^{\alpha}\right\}$ of a generic layer $i$ is given by:

$$
\begin{gathered}
u_{1}\left(z^{1}, z^{2}, z^{3}\right)=u_{o 1}\left(z^{1}, z^{2}\right)-z^{3} \beta_{1}\left(z^{1}, z^{2}\right) \\
u_{2}\left(z^{1}, z^{2}, z^{3}\right)=u_{o 2}\left(z^{1}, z^{2}\right)-z^{3} \beta_{2}\left(z^{1}, z^{2}\right) \\
u_{3}\left(z^{1}, z^{2}, z^{3}\right)=w\left(z^{1}, z^{2}\right)
\end{gathered}
$$

where, $\boldsymbol{u}=\left[u_{o 1}, u_{o 2}\right]^{\mathrm{T}}$ are axial displacements in the middle surface of the layer, $\boldsymbol{\beta}=\left[\beta_{1}, \beta_{2}\right]^{\mathrm{T}}$ are rotations of the transverse (assumed inextensible) normal around the axes $z^{2}, z^{1}$, respectively, and $w$ is the constant transverse displacements in the thickness.

The joint behavior of the sheets that make up the 
multilayer system necessarily supposes the continuity of displacements between adjacent layers (Fig. 1), for which it is imposed:

(1) The displacements in the plane of the lower fibers of the top layer $i$ must be equal to those of the upper fibers of the bottom plate $i+1$ :

$$
\begin{aligned}
& u_{1}^{\langle i\rangle}\left(z^{1}, z^{2},+\frac{h_{i}}{2}\right)=u_{1}^{\langle i+1\rangle}\left(z^{1}, z^{2},-\frac{h_{i+1}}{2}\right) \\
& u_{2}^{\langle i\rangle}\left(z^{1}, z^{2},+\frac{h_{i}}{2}\right)=u_{2}^{\langle i+1\rangle}\left(z^{1}, z^{2},-\frac{h_{i+1}}{2}\right)
\end{aligned}
$$

(2) The transverse displacements in the upper layer $i$ must be the same as those of the lower plate $i+1$ :

$$
w^{\langle i\rangle}\left(z^{1}, z^{2}\right)=w^{\langle i+1\rangle}\left(z^{1}, z^{2}\right)
$$

The functional of the total potential energy of the multilayer system is presented as an superposition of $n$ simple plates where the kinematic constraints are mathematically ensured from the incorporation of Lagrange multipliers $\lambda^{\langle j\rangle}=\left[\lambda_{1}^{\langle j\rangle}, \lambda_{2}^{\langle j\rangle}\right]^{\mathrm{T}}$ and $\boldsymbol{\mu}^{\langle j\rangle}$ which represent the static variables at interface $j$ (interlaminar transverse stresses):

$$
\begin{gathered}
\Pi=\sum_{i=1}^{n}\left\{\frac{1}{2} h_{i} a\left(\boldsymbol{u}^{\langle i\rangle}, \boldsymbol{u}^{\langle i\rangle}\right)+\frac{1}{2} \frac{h_{i}{ }^{3}}{12} a\left(\boldsymbol{\beta}^{\langle i\rangle}, \boldsymbol{\beta}^{\langle i\rangle}\right)+\frac{1}{2} h_{i} b\left(\nabla w^{\langle i\rangle}-\boldsymbol{\beta}^{\langle i\rangle}, \nabla w^{\langle i\rangle}-\boldsymbol{\beta}^{\langle i\rangle}\right)-W_{e x t}^{\langle i\rangle}\right\}+ \\
+\sum_{j=1}^{n-1}\left\{\int_{S^{\langle j+1\rangle}} \lambda^{\langle j+1\rangle \mathrm{T}} \cdot\left[\left(\boldsymbol{u}^{\langle j\rangle}-\frac{h_{j}}{2} \boldsymbol{\beta}^{\langle j\rangle}\right)-\left(\boldsymbol{u}^{\langle j+1\rangle}+\frac{h_{j+1}}{2} \boldsymbol{\beta}^{\langle j+1\rangle}\right)\right] d \Omega++\int_{S^{\langle j+1\rangle}}\left(w^{\langle j\rangle}-w^{\langle j+1\rangle}\right) \mu^{\langle j+1\rangle} d \Omega\right\}
\end{gathered}
$$
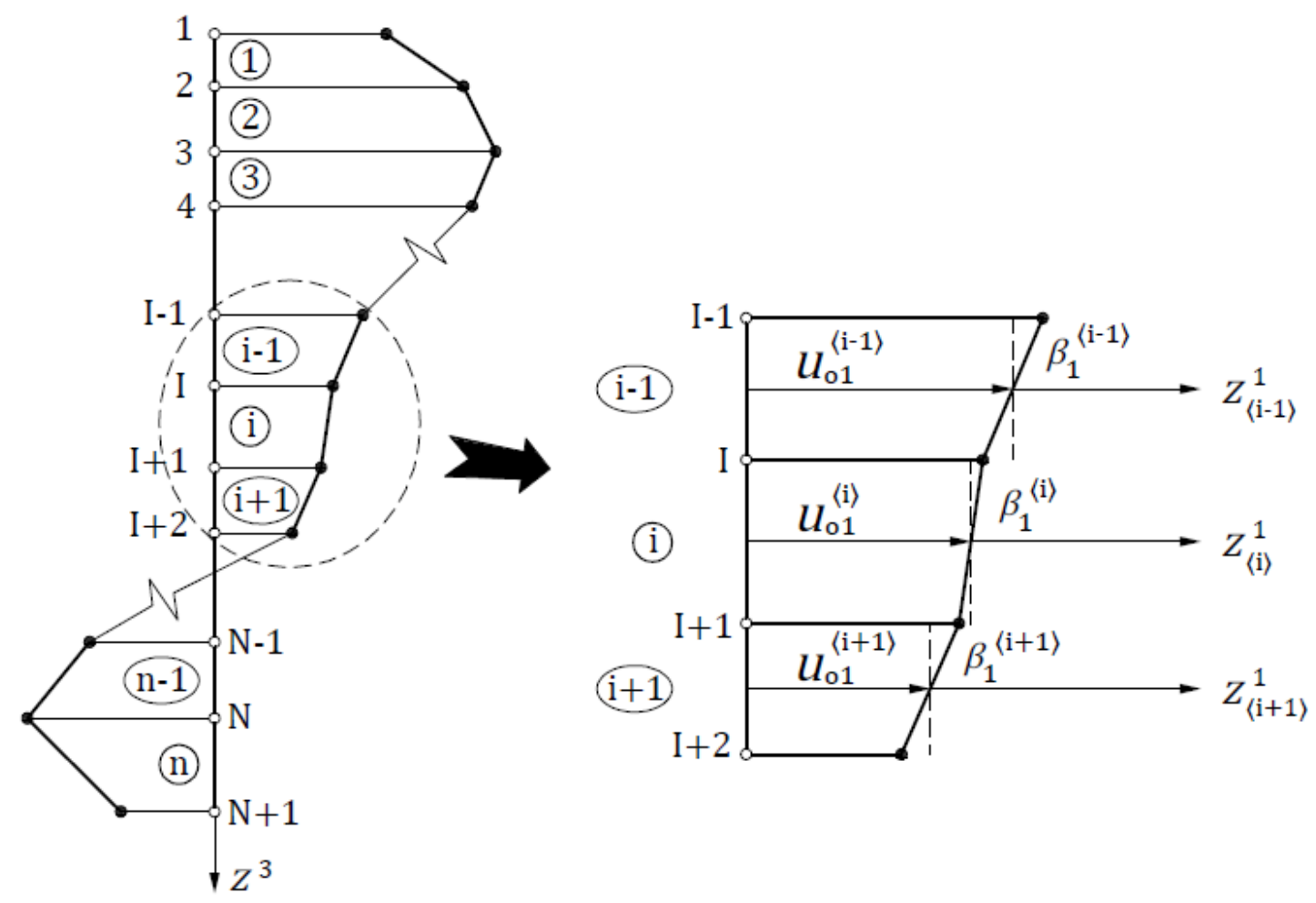

Fig. 1 Displacements field through the thickness of the multilayer plate. 
where, $a\left(\boldsymbol{u}^{\langle i\rangle}, \boldsymbol{u}^{\langle i\rangle}\right)$ and $a\left(\boldsymbol{\beta}^{\langle i\rangle}, \boldsymbol{\beta}^{\langle i\rangle}\right)$ are the membrane and flexural energy for each layer, respectively, while $b\left(\boldsymbol{\nabla} w^{\langle i\rangle}-\boldsymbol{\beta}^{\langle i\rangle}, \boldsymbol{\nabla} w^{\langle i\rangle}-\boldsymbol{\beta}^{\langle i\rangle}\right)$ is the deformation shear energy. The total external work in each layer is named $W_{\text {ext }}^{\langle i\rangle}$. Only the work of the forces in the plane is considered due to the transversal deflection:

$$
W_{e x t}^{\langle i\rangle}=\int_{\Omega_{i}} \frac{1}{2} \nabla w^{\langle i\rangle \mathrm{T}} \cdot \mathbf{N}^{\langle i\rangle} \cdot \nabla w^{\langle i\rangle} d \Omega
$$

with,

$$
\begin{aligned}
& \mathbf{N}^{\langle i\rangle}=\left[\begin{array}{ll}
\eta_{11}^{\langle i\rangle} \mathrm{N}_{11} & \eta_{12}^{\langle i\rangle} \mathrm{N}_{12} \\
\eta_{22}^{\langle i\rangle} \mathrm{N}_{22} & \eta_{22}^{\langle i\rangle} \mathrm{N}_{22}
\end{array}\right] \\
& \boldsymbol{\nabla} w^{\langle i\rangle}=\left[\begin{array}{ll}
w_{1}{ }_{1}^{\langle i\rangle} & w_{2},_{2}^{\langle i\rangle}
\end{array}\right]^{\mathrm{T}} \\
& \delta \boldsymbol{u}^{\langle i\rangle}: h_{i} a\left(\boldsymbol{u}^{\langle i\rangle}, \delta \boldsymbol{u}^{\langle i\rangle}\right)-\int \delta \boldsymbol{u}^{\langle i\rangle \mathrm{T}} \cdot \lambda^{\langle i\rangle} d \Omega+\int \delta \boldsymbol{u}^{\langle i\rangle \mathrm{T}} \cdot \lambda^{\langle i+1\rangle} d \Omega=0 \\
& \delta w^{\langle i\rangle}: h_{i} b\left(\nabla w^{\langle i\rangle}-\boldsymbol{\beta}^{\langle i\rangle}, \delta \nabla w^{\langle i\rangle}\right)-\int_{\Omega_{i}} \delta w^{\langle i\rangle} \mu^{\langle i\rangle} d \Omega+\int_{\Omega_{i}} \delta w^{\langle i+1\rangle} \mu^{\langle i+1\rangle} d \Omega \\
& -\int_{\Omega_{i}} \delta \nabla w^{\langle i\rangle \mathrm{T}} \cdot \mathbf{N}^{\langle i\rangle} \cdot \nabla w^{\langle i\rangle} d \Omega=0 \\
& \delta \boldsymbol{\beta}^{\langle i\rangle}: \frac{h_{i}^{3}}{12} a\left(\boldsymbol{\beta}^{\langle i\rangle}, \delta \boldsymbol{\beta}^{\langle i\rangle}\right)+h_{i} b\left(\nabla w^{\langle i\rangle}-\boldsymbol{\beta}^{\langle i\rangle},-\delta \boldsymbol{\beta}^{\langle i\rangle}\right)+ \\
& -\int_{\Omega_{i}} \frac{h_{i}}{2} \delta \boldsymbol{\beta}^{\langle i\rangle \mathrm{T}} \cdot \lambda^{\langle i\rangle} d \Omega-\int_{\Omega_{i}} \frac{h_{i}}{2} \delta \boldsymbol{\beta}^{\langle i\rangle \mathrm{T}} \cdot \lambda^{\langle i\rangle} d \Omega=0 \\
& \delta \boldsymbol{\lambda}^{\langle j\rangle}: \int_{S^{\langle j+1\rangle}} \delta \boldsymbol{\lambda}^{\langle j+1\rangle \mathrm{T}} \cdot\left[\left(\boldsymbol{u}^{\langle j-1\rangle}-\frac{h_{j-1}}{2} \boldsymbol{\beta}^{\langle j-1\rangle}\right)-\left(\boldsymbol{u}^{\langle j+1\rangle}+\frac{h_{j}}{2} \boldsymbol{\beta}^{\langle j\rangle}\right)\right] d \Omega=0 \\
& \delta \mu^{\langle j\rangle}: \int_{S^{\langle j+1\rangle}}\left(w^{\langle j-1\rangle}-w^{\langle j\rangle}\right) \delta \mu^{\langle j\rangle} d \Omega=0
\end{aligned}
$$

where, $i=1, \ldots, n ; j=2, \ldots, n$. The Greek letter $\delta$ symbolizes the variational operator.

The kinematic variables require continuity $\mathrm{C}^{0}$ when only their first derivatives appear in the Eqs. (8)-(12),

$$
\left(\boldsymbol{u}^{\langle i\rangle}, \boldsymbol{w}^{\langle i\rangle}, \boldsymbol{\beta}^{\langle i\rangle}, \boldsymbol{\lambda}^{\langle j\rangle}, \mu^{\langle j\rangle}, \mathbf{z}\right)=\sum_{k=1}^{N N} N_{k}\left(\widehat{\boldsymbol{u}}^{k\langle i\rangle}, \widehat{w}^{k\langle i\rangle}, \widehat{\boldsymbol{\beta}}^{k\langle i\rangle}, \hat{\boldsymbol{\lambda}}^{k\langle j\rangle}, \widehat{\mu}^{k\langle j\rangle}, \widehat{\mathbf{z}}_{k}\right)
$$

where, $N N$ represents the number of nodes of the element and $N_{k}$ are the interpolation functions associated with node $k$. The circumflex tilde $\left(^{\wedge}\right)$ whereas the variables can be discontinuous (continuity $\mathrm{C}^{-1}$ ). However, the same interpolation is used for all the unknowns for simplicity.

Using isoparametric finite elements [3], indicates nodal variable.

Incorporating Eq. (13) into Eqs. (8)-(12) gives the finite element model: 


$$
\begin{gathered}
\delta \widehat{\boldsymbol{u}}^{\langle i\rangle \mathrm{T}} \cdot\left[\mathbf{A}_{m}^{\langle i\rangle} \cdot \widehat{\boldsymbol{u}}^{\langle i\rangle}-\mathbf{B}_{i}^{\langle i+1\rangle} \cdot \hat{\boldsymbol{\lambda}}^{\langle i+1\rangle}+\mathbf{B}_{i}^{\langle i\rangle} \cdot \hat{\boldsymbol{\lambda}}^{\langle i\rangle}\right]=0 \\
\delta \widehat{\boldsymbol{w}}^{\langle i\rangle \mathrm{T}} \cdot\left[\mathbf{G}^{\langle i\rangle} \cdot \widehat{\boldsymbol{w}}^{\langle i\rangle}-\mathbf{E}^{\langle i\rangle \mathrm{T}} \cdot \widehat{\boldsymbol{\beta}}^{\langle i\rangle}-\mathbf{H}_{i}^{\langle i\rangle} \cdot \widehat{\boldsymbol{\mu}}^{\langle i\rangle}-\mathbf{H}_{i}^{\langle i+1\rangle} \cdot \widehat{\boldsymbol{\mu}}^{\langle i+1\rangle}-\Lambda \mathbf{k}_{g}^{\langle i\rangle} \cdot \widehat{\boldsymbol{w}}^{\langle i\rangle}\right]=0 \\
\delta \widehat{\boldsymbol{\beta}}^{\langle i\rangle \mathrm{T}} \cdot\left[\mathbf{A}_{b}^{\langle i\rangle} \cdot \widehat{\boldsymbol{\beta}}^{\langle i\rangle}-\mathbf{E}^{\langle i\rangle} \cdot \widehat{\boldsymbol{w}}^{\langle i\rangle}+\mathbf{D}^{\langle i\rangle} \cdot \widehat{\boldsymbol{\beta}}^{\langle i\rangle}-\mathbf{C}_{i}^{\langle i\rangle} \cdot \hat{\boldsymbol{\lambda}}^{\langle i\rangle}-\mathbf{C}_{i}^{\langle i+1\rangle} \cdot \widehat{\boldsymbol{\lambda}}^{\langle i+1\rangle}\right]=0 \\
\delta \hat{\boldsymbol{\lambda}}^{\langle j\rangle \mathrm{T}} \cdot\left[\mathbf{B}_{j-1}^{\langle j\rangle \mathrm{T}} \cdot \widehat{\boldsymbol{u}}^{\langle j-1\rangle}-\mathbf{C}_{j-1}^{\langle j\rangle \mathrm{T}} \cdot \widehat{\boldsymbol{\beta}}^{\langle j-1\rangle}-\mathbf{B}_{j}^{\langle j\rangle \mathrm{T}} \cdot \widehat{\boldsymbol{u}}^{\langle j\rangle}-\mathbf{C}_{j}^{\langle j\rangle \mathrm{T} \mathrm{T}} \cdot \widehat{\boldsymbol{\beta}}^{\langle j\rangle}\right]=0 \\
\delta \widehat{\boldsymbol{\mu}}^{\langle j\rangle \mathrm{T} \mathrm{T}} \cdot\left[\mathbf{H}_{j-1}^{\langle j\rangle \mathrm{T}} \widehat{\boldsymbol{w}}^{\langle j-1\rangle}-\mathbf{H}_{j}^{\langle j\rangle \mathrm{T}} \widehat{\boldsymbol{w}}^{\langle j\rangle}\right]=0
\end{gathered}
$$

For a 3-layer system, the matrix representation in compact form is presented below:

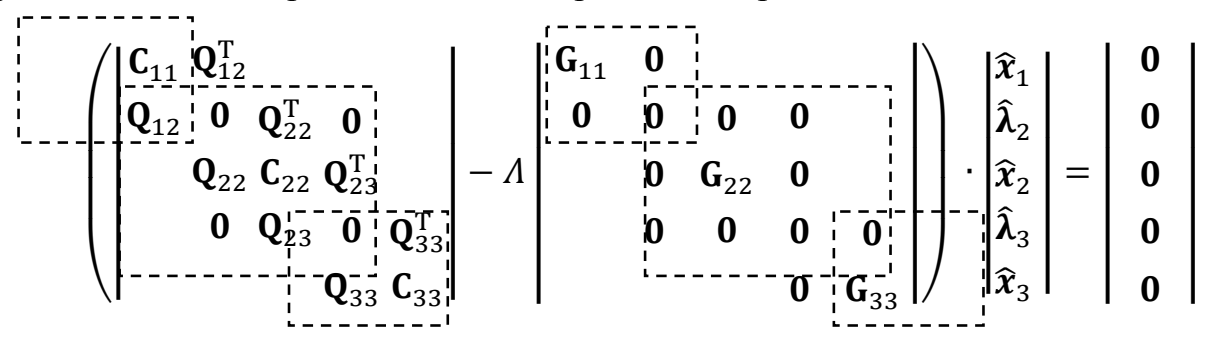

where, $\widehat{\boldsymbol{x}}_{i}$ are the nodal kinematic variables, $\hat{\boldsymbol{\lambda}}_{i}$ are the nodal static variables, $\left(\mathbf{C}_{i i}, \mathbf{G}_{i i}\right)$ are the contributions of mechanical and geometric rigidity of each layer, respectively, and $\left(\mathbf{Q}_{i i}, \mathbf{Q}_{i j}\right)$ are the matrices resulting from the constraint Lagrangian problem. The factor $\Lambda$ is the critical buckling load parameter, defined as:

$$
\Lambda=\frac{\mathrm{N}_{c r}}{\mathrm{~N}_{11}}=\frac{\mathrm{N}_{c r}}{\mathrm{~N}_{22}}=\frac{\mathrm{N}_{c r}}{\mathrm{~N}_{12}}
$$

The no trivial solution from Eq. (10) requires:

$$
\operatorname{det}\left(\mathbf{K}-\Lambda \mathbf{K}_{\mathbf{G}}\right)=0
$$

after applying the boundering conditions.

\section{Eigen Value Problem Solution}

As is characteristic of mixed formulations, the

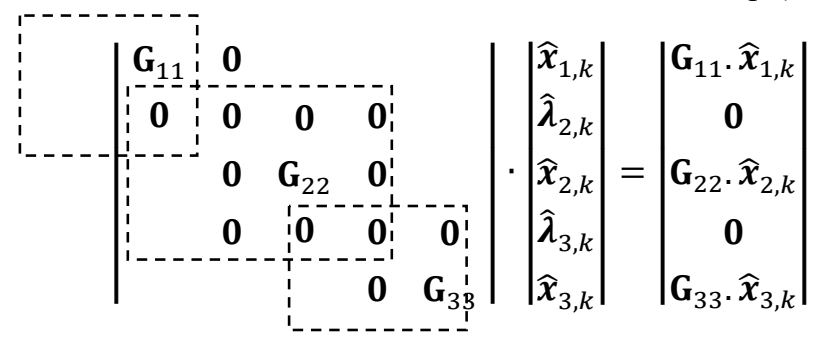

Since the initial vector is arbitrarily defined, Eq. (19) is generally not true (if satisfied, the test vector is an mechanical stiffness matrix is not positively definite, nor is the geometric stiffness matrix. In addition, the number of unknowns increases rapidly as more layers are added to the model, so that the determination of the eigenvalue problem is difficult. In this section, a simple solution methodology is proposed based on the inverse iteration [4].

The procedure is to initially establish a test vector. Subsequently, the term involving the geometric stiffness matrix is evaluated. Since the eigenvectors can only be determined within a scale factor, the choice $\Lambda$ does not affect the result, so it is assumed $\Lambda=1$. In this way, it is obtained the equivalent load vector shown in Eq. (22).

eigenvector), so the following system of equations must be solved: 


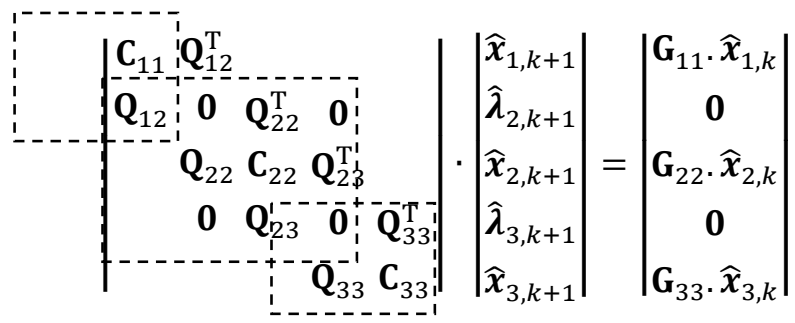

From Eq. (23), it results:

$$
\begin{gathered}
\mathbf{C}_{11} \widehat{\boldsymbol{x}}_{1, k+1}=\mathbf{G}_{11} \widehat{\boldsymbol{x}}_{1, k}-\mathbf{Q}_{12}^{\mathrm{T}} \hat{\boldsymbol{\lambda}}_{2, k+1} \\
\mathbf{C}_{22} \widehat{\boldsymbol{x}}_{2, k+1}=\mathbf{G}_{22} \widehat{\boldsymbol{x}}_{2, k}-\mathbf{Q}_{22} \hat{\lambda}_{2, k+1}-\mathbf{Q}_{23}^{\mathrm{T}} \hat{\lambda}_{3, k+1} \\
\mathbf{C}_{33} \widehat{\boldsymbol{x}}_{3, k+1}=\mathbf{G}_{33} \widehat{\boldsymbol{x}}_{3, k}-\mathbf{Q}_{33} \hat{\boldsymbol{\lambda}}_{3, k+1}
\end{gathered}
$$

Replacing the displacements in the remaining equations yields the reduced matrix shown in Eq. (25):

$$
\begin{gathered}
\left|\begin{array}{cc}
\mathbf{Q}_{12} \cdot \mathbf{C}_{11}^{-1} \cdot \mathbf{Q}_{12}^{\mathrm{T}}+\mathbf{Q}_{22}^{\mathrm{T}} \cdot \mathbf{C}_{22}^{-1} \cdot \mathbf{Q}_{22} & \mathbf{Q}_{22}^{\mathrm{T}} \cdot \mathbf{C}_{22}^{-1} \cdot \mathbf{Q}_{23}^{\mathrm{T}} \\
\mathbf{Q}_{23} \cdot \mathbf{C}_{22}^{-1} \cdot \mathbf{Q}_{22} & \mathbf{Q}_{23} \cdot \mathbf{C}_{22}^{-1} \cdot \mathbf{Q}_{23}^{\mathrm{T}}+\mathbf{Q}_{33}^{\mathrm{T}} \cdot \mathbf{C}_{33}^{-1} \cdot \mathbf{Q}_{33}
\end{array}\right| \cdot\left|\begin{array}{l}
\hat{\boldsymbol{\lambda}}_{2, k+1} \\
\hat{\boldsymbol{\lambda}}_{3, k+1}
\end{array}\right|= \\
=\left|\begin{array}{l}
\mathbf{Q}_{12} \cdot \mathbf{C}_{11}^{-1} \cdot \mathbf{G}_{11} \cdot \widehat{\boldsymbol{x}}_{1, k}-\mathbf{Q}_{22}^{\mathrm{T}} \cdot \mathbf{C}_{11}^{-1} \cdot \mathbf{G}_{22} \cdot \widehat{\boldsymbol{x}}_{2, k} \\
\mathbf{Q}_{23} \cdot \mathbf{C}_{22}^{-1} \cdot \mathbf{G}_{22} \cdot \widehat{\boldsymbol{x}}_{2, k}-\mathbf{Q}_{33}^{\mathrm{T}} \cdot \mathbf{C}_{33}^{-1} \cdot \mathbf{G}_{33} \cdot \widehat{\boldsymbol{x}}_{3, k}
\end{array}\right|
\end{gathered}
$$

Thus, the kinematic variables are determined from the static variables, solving the "mixed problem".

The sufficient but not necessary condition for solving the system Eq. (25) results:

$$
n_{x_{i}} \geq n_{\lambda_{i}}
$$

where, $n_{x_{i}}$ and $n_{\lambda_{i}}$ are the number of degrees of freedom of the corresponding variables [5].

The correspondent eigenvalue is computed applying the Rayleigh Coefficient:

$$
\Lambda_{k+1}=\sum_{i=1}^{n} \frac{\widehat{\boldsymbol{x}}_{i, k+1}^{\mathrm{T}} \cdot \mathbf{G}_{i i} \cdot \widehat{\boldsymbol{x}}_{i, k}}{\widehat{\boldsymbol{x}}_{i_{i, k+1} \mathrm{~T}}^{\mathrm{T}} \cdot \mathbf{G}_{i i} \cdot \widehat{\boldsymbol{x}}_{i, k+1}}
$$

Then the resultant vector is normalized in order that the new vector satisfies:

$$
\sum_{i=1}^{n} \widehat{\boldsymbol{x}}_{i, k+1}^{T} \cdot \mathbf{G}_{i i} \cdot \widehat{\boldsymbol{x}}_{i_{, k+1}}=1
$$

Normalization maintains vector elements with similar values during each iteration. If it is not done, the values of the elements grow and decrease at each step, which can cause numeric problems.

The convergence check is specified by comparing two successive values of the eigenvalue:

$$
\left|\frac{\Lambda_{k+1}-\Lambda_{k}}{\Lambda_{k+1}}\right| \leq \text { tolerancia }
$$

If the convergence criterion is not satisfied, a new iteration is started using the test vector obtained when solving the system Eq. (23). The procedure is repeated until the established convergence is reached. As the number of iterations increases, the eigenvalue and eigenvector are lowered.

\section{Numerican Results}

From the presented model, a code is implemented in the GNU Octave program for the determination of critical loads of composite plate buckling. The effects of the material and the thickness/side ratio on the collapse load are studied. In addition, the variation through the thickness of the transverse stresses associated with the buckling mode is presented.

The analyzed plates have a rectangular geometry with dimensions $a, b$ coincident with the $x, y$ directions respectively (see Fig. 2). In all the examples, for reasons of symmetry, only one quarter of a plate is modeled with a mesh of $6 \times 6$ quadrilateral four-node isoparametric elements with linear interpolation. The numerical evaluation of the integrals is carried out using the Gauss quadrature method, using $2 \times 2$ points 


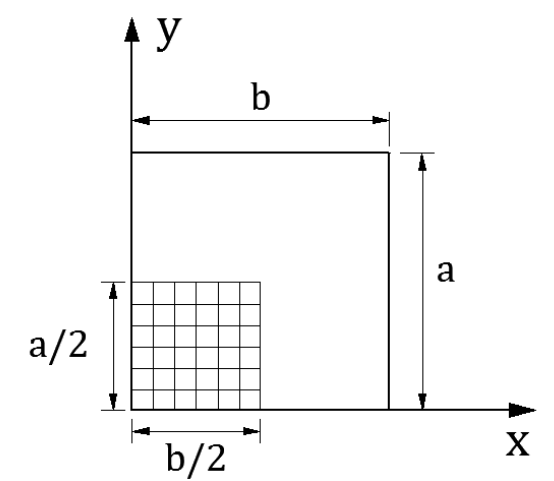

Fig. 2 Plate representation and typical mesh used in the model.

for the membrane terms, flexion, while $1 \times 1$ points for geometry and geometric stiffness. The tolerance to accept the solution in the problem of eigenvalues was established in 1e-6.

The edge conditions used are:

$$
\begin{gathered}
(x, 0): w=u_{o x}=\beta_{x}=0 \\
(0, y): w=u_{o y}=\beta_{y}=0 \\
(x, b / 2): u_{o y}=\beta_{y}=0 \\
(a / 2, y): u_{o x}=\beta_{x}=0
\end{gathered}
$$

The elastic properties of the materials are:

$$
\frac{G_{23}}{E_{2}}=0.6 \quad \frac{G_{12}}{E_{2}}=\frac{G_{13}}{E_{2}}=0.5 \quad v_{12}=0.25
$$

where, the ratio E1/E2 is specific for each particular example. The value of E2 is arbitrary because of the dimensionlessness used:

$$
\overline{\mathrm{N}}_{c r}=\Lambda \mathbf{N} \frac{a^{2}}{E_{2} h^{3}}
$$

Table 1 shows the uni-axial critical buckling load for a laminated square plate $\left(0^{\circ} / 90^{\circ} / 0^{\circ}\right)$ with a thickness/side $h / a=0.10$ ratio and different E1/E2 ratios. The obtained results are contrasted with the solution of the elasticity 3D5, noting an excellent correlation. It is noted that as the number of calculation layers is increased, the solution presented is refined as a direct consequence of computing more realistically strain energy by cutting through the thickness, which is assumed constant in each layer.

The effect of the thickness/side ratio on the critical buckling load is studied in Table 2. A laminated square plate $\left(0^{\circ} / 90^{\circ}\right)$ with an E1/E2 $=40$ ratio under uni-axial loading is analyzed. Each individual sheet was discretized in four calculation layers. The results are compared with those of other authors.

Finally, the physical meaning of the Lagrange multipliers is evident. The variation along the thickness of the transverse interlaminar stresses associated with the failure mode in Fig. 3 for the plate of the first example with relation E1/E2 $=40$ is plotted, while in Fig. 4 for the plate of the second case with ratio $h / a=$ 0.10 . The values obtained are divided by their maximum absolute value. The distribution obtained is

Table1 Individual layer orthotropic degree effect on the buckling axial critic load of a square plate $\left(0^{\circ} / 90^{\circ} / 0^{\circ}\right)$, simple support with $h / a=0.10$.

\begin{tabular}{lllllll}
\hline \multirow{2}{*}{ Reference } & \multirow{2}{*}{ Thickness discretization } & \multicolumn{5}{c}{ Relation $\mathrm{E}_{1} / \mathrm{E}_{2}$} \\
\cline { 3 - 7 } & & 3 & 10 & 20 & 30 & 40 \\
\hline \multirow{2}{*}{ Current } & 3 layers & 5.4972 & 10.0842 & 15.3656 & 19.5699 & 23.0107 \\
& 9 layers & 5.4720 & 9.9811 & 15.1017 & 19.1299 & 22.3981 \\
& 15 layers & 5.4698 & 9.9720 & 15.0782 & 19.0902 & 22.3418 \\
\hline Noor [6] & 5.3044 & 9.7621 & 15.0191 & 19.3040 & 22.8807 \\
\hline Singh et al. [7] & 5.2284 & 9.6259 & 14.6458 & 18.6158 & 21.8527 \\
\hline Reddy and Pan [8] & 5.3933 & 9.9406 & 15.2980 & 19.6740 & 22.3400 \\
\hline
\end{tabular}

Table 2 Buckling axial critic load for a square laminated plate $\left(0^{\circ} / 90^{\circ}\right)$, simple support varying $h / a$.

\begin{tabular}{llllll}
\hline Relation $h / a$ & Singh et al. [7] & Reddy and Pan [8] & Van et al. [9] & $\begin{array}{l}\text { Chakrabarti and } \\
\text { Sheikh [10] }\end{array}$ & Current \\
\hline 0.10 & 11.310 & 11.563 & 11.360 & 11.349 & 11.275 \\
0.05 & 12.427 & 12.577 & 12.551 & 12.510 & 12.642 \\
0.02 & 12.800 & 12.895 & 12.906 & 12.879 & 13.091 \\
0.01 & 12.873 & 12.942 & 13.039 & 12.934 & 13.158 \\
\hline
\end{tabular}



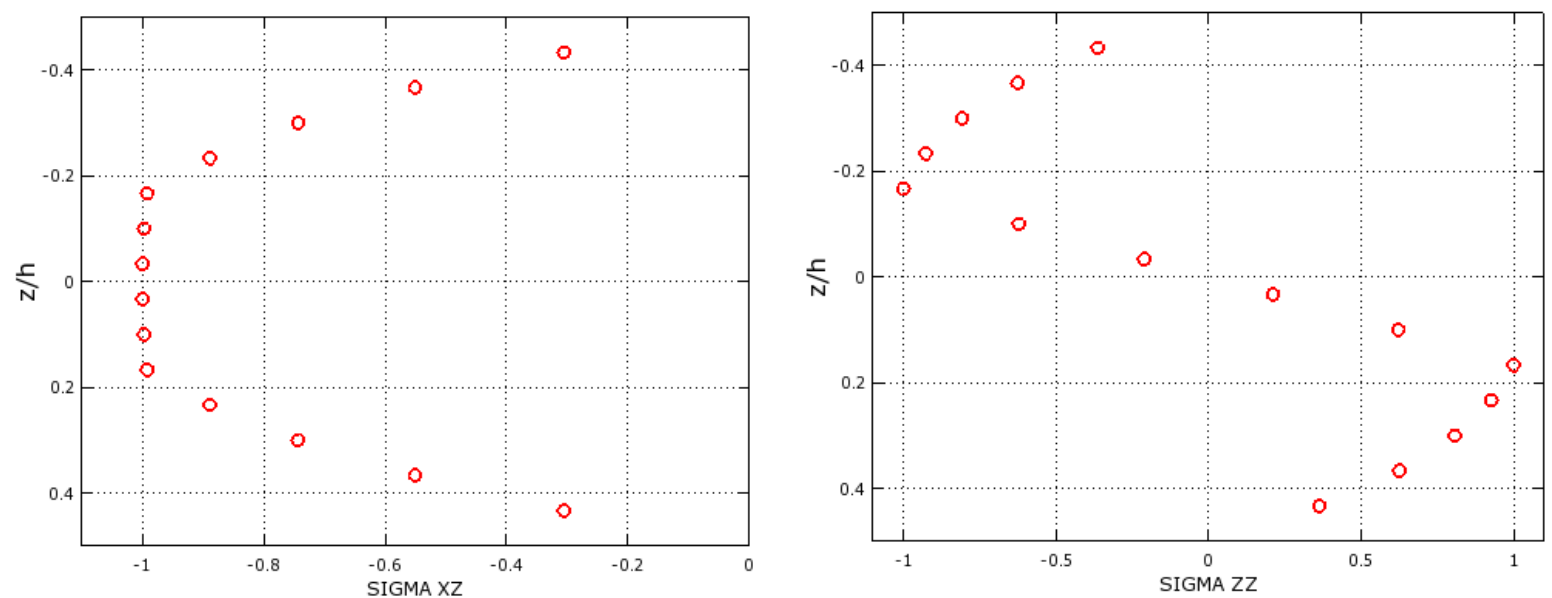

Fig. 3 Transversal shear stress variation through the depth $\bar{\sigma}_{13}\left(0, \frac{b}{2}, \bar{z}^{3}\right)$ and transversal norlam stress $\bar{\sigma}_{33}\left(\frac{a}{2}, \frac{b}{2}, \bar{z}^{3}\right)$ normalized for a square laminated plate $\left(0^{\circ} / 90^{\circ}\right)$ simple supported $\left(h / a=0.10-E_{1} / E_{2}=40-16\right.$ layers $)$ : (a) XZ; (b) ZZ.
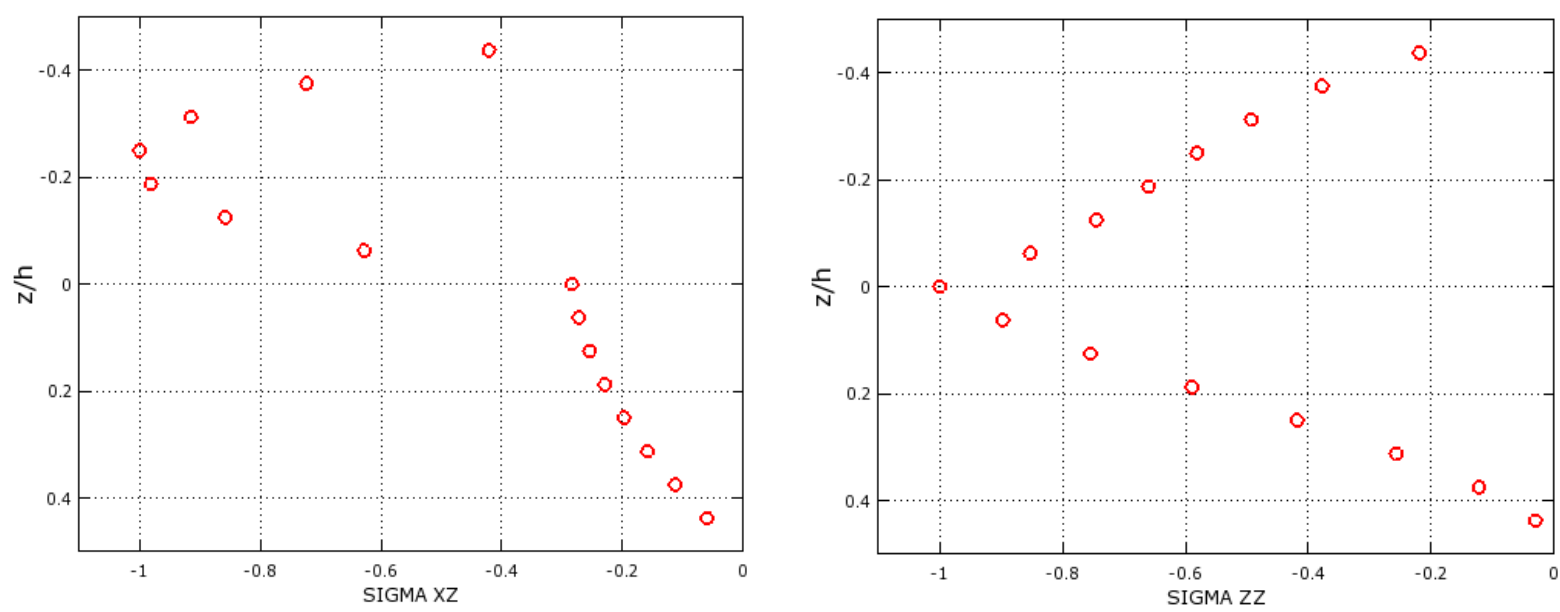

Fig. 4 Transversal shear stress variation through the depth $\bar{\sigma}_{13}\left(0, \frac{b}{2}, \overline{\mathbf{z}}^{3}\right)$ and transversal norlam stress $\bar{\sigma}_{33}\left(\frac{a}{2}, \frac{b}{2}, \bar{z}^{3}\right)$ normalized for a square laminated plate $\left(0^{\circ} / 90^{\circ}\right)$ simple supported $\left(h / a=0.10-E_{1} / E_{2}=40-16\right.$ layers $)$ : (a) XZ; (b) ZZ.

as expected, with the contour conditions at the upper and lower edges being met.

\section{Conclusions}

A mixed finite element model is presented to determine the critical buckling load of multilayer plates. The continuity of the displacements as well as the transverse stresses is ensured along the thickness of the plate from the inclusion of Lagrange multipliers. The mixed formulation allows a direct evaluation of the transverse stresses without the need to integrate the equilibrium equations that imply losses of precision, resulting in a point of great interest in the study of the response of composite laminates. A methodology is implemented from the inverse iteration method to solve the problem of eigenvalues with satisfactory results. The accuracy of the model is demonstrated along a series of problems where the results found resemble those available in the literature.

\section{References}

[1] Alliney, S., and Carnicer, R. S. 1992. "A Hybrid Finite Element Model for Multilayered Plates." Computational Mechanics 10 (5): 319-33.

[2] Carnicer, R. S., Desía, B. A., Schwarz, R. A., and Pan, Q. 2012. "Análisis de Places Multicapas Mediante un Modelo de Elementos Finitos Híbrido.” Mecánica Computacional XXXI: 857-74. (in Galician) 
[3] Bathe, K. J. 1996. Finite Element Procedures. New Jersey: Prentice Hall.

[4] Chopra, A. K. 1996. Dynamics of Structures: Theory and Applications to Earthquake Engineering. New Jersey: Prentice Hall, 392-405.

[5] Zienkiewicz, O. C., and Taylor, R. L. 1994. El Método de los Elementos Finitos: Formulaciones Básicas y Problemas Lineales. Madrid: McGraw Hill, 333-87. (in Spanish)

[6] Noor, A. K. 1975. "Stability of Multilayered Composite Plates." Fibre Science and Technology 8 (2): 81-9.

[7] Singh, S. K., and Chakrabarti, A. 2012. "Buckling Analysis of Laminated Composite Plates Using an Efficient $\mathrm{C}^{0}$ FE Model." Latin American Journal of Solids and Structures 9: 353-65.

[8] Reddy, J. N., and Phan, N. D. 1985. "Stability and Vibration of Isotropic, Orthotropic and Laminated Plates According to a Higher-Order Shear Deformation Theory." Journal of Sound and Vibrations 98 (2): 157-70.

[9] Nguayen-Van, H., Mai-Duy, N., Karanasena, W., and Tran-Cong, T. 2011. "Buckling and Vibration Analysis of Laminated Composite Plate/Shell Structures via a Smoothed Flat Shell Element with In-plane Rotations." Composite Structures 89: 612-25.

[10] Chakrabarti, A., and Sheikh, H. 2003. "Buckling of Laminated Composite Plates by a New Element Based on Higher Order Shear Deformation Theory." Mechanic of Composite Materials and Structures 10 (4): 303-17. 


\section{Appendix A: Buckling Coefficients}

When each layer is modeled independently, it is necessary to distribute the critical buckling load of the plate in the different layers. For this purpose, the set of layers is considered as a system of springs in parallel (see Fig. 5).
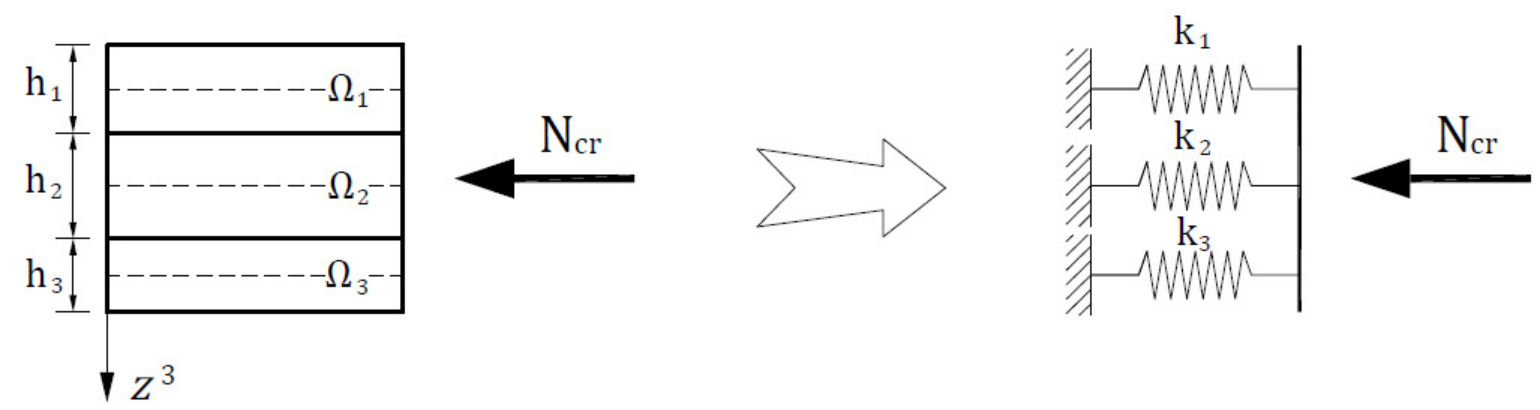

Fig. 5 Load distribution by layer: parallel spring analogy.

For a general layer,

$$
\begin{gathered}
\varepsilon_{11}^{o}=\frac{1}{h}\left(s_{11} \mathrm{~N}_{11}+s_{12} \mathrm{~N}_{22}+s_{16} \mathrm{~N}_{12}\right) \\
\varepsilon_{22}^{o}=\frac{1}{h}\left(s_{12} \mathrm{~N}_{11}+s_{22} \mathrm{~N}_{22}+s_{26} \mathrm{~N}_{12}\right) \\
\varepsilon_{12}^{o}=\frac{1}{h}\left(s_{16} \mathrm{~N}_{11}+s_{26} \mathrm{~N}_{22}+s_{66} \mathrm{~N}_{12}\right)
\end{gathered}
$$

where, $\left(\varepsilon_{11}^{o}, \varepsilon_{22}^{o}, \varepsilon_{12}^{o}\right)$ are the membranal strains in the layer plane, while $s_{\alpha \gamma}(\alpha, \gamma=1,2,6)$ are the coefficients of the inverse marix of the elastic coefficients.

From Eq. (33),

$$
\begin{aligned}
\mathrm{N}_{11} & =\frac{h}{s_{11}+a_{11} s_{12}+b_{11} s_{16}} \varepsilon_{11}^{o} \\
\mathrm{~N}_{22} & =\frac{h}{a_{22} s_{12}+s_{22}+b_{22} s_{26}} \varepsilon_{22}^{o} \\
\mathrm{~N}_{12} & =\frac{h}{a_{12} s_{16}+b_{12} s_{26}+s_{66}} \varepsilon_{12}^{o}
\end{aligned}
$$

where,

$$
\begin{array}{cc}
a_{11}=\frac{\mathrm{N}_{22}}{\mathrm{~N}_{11}} & b_{11}=\frac{\mathrm{N}_{12}}{\mathrm{~N}_{11}} \\
a_{22}=\frac{\mathrm{N}_{11}}{\mathrm{~N}_{22}} & b_{22}=\frac{\mathrm{N}_{12}}{\mathrm{~N}_{22}} \\
a_{12}=\frac{\mathrm{N}_{11}}{\mathrm{~N}_{12}} & b_{12}=\frac{\mathrm{N}_{22}}{\mathrm{~N}_{12}}
\end{array}
$$

Assuming that the springs (layers) have the same deformation, after some algebra, we get:

$$
\begin{aligned}
& \eta_{11}^{\langle i\rangle}=\frac{\mathrm{N}_{11}^{\langle i\rangle}}{\mathrm{N}_{11}}=\frac{h_{i}}{s_{11}^{\langle i\rangle}+a_{11}^{\langle i\rangle} s_{12}^{\langle i\rangle}+b_{11}^{\langle i\rangle} s_{16}^{\langle i\rangle}} \sum_{i=1}^{n}\left\{\frac{s_{11}^{\langle i\rangle}+a_{11}^{\langle i\rangle} s_{12}^{\langle i\rangle}+b_{11}^{\langle i\rangle} s_{16}^{\langle i\rangle}}{h_{i}}\right\} \\
& \eta_{22}^{\langle i\rangle}=\frac{\mathrm{N}_{22}^{\langle i\rangle}}{\mathrm{N}_{22}}=\frac{h_{i}}{a_{22}^{(i)} s_{12}^{\langle i\rangle}+s_{22}^{\langle i}+b_{22}^{\langle i\rangle} s_{26}^{i\rangle}} \sum_{i=1}^{n}\left\{\frac{a_{22}^{\langle i\rangle} s_{12}^{\langle i\rangle}+s_{22}^{\langle i\rangle}+b_{22}^{\langle i\rangle} s_{26}^{\langle i\rangle}}{h_{i}}\right\} \\
& \eta_{12}^{\langle i\rangle}=\frac{\mathrm{N}_{12}^{\langle i\rangle}}{\mathrm{N}_{22}}=\frac{h_{i}}{a_{12}^{\langle i\rangle} s_{16}^{\langle i\rangle}+b_{12}^{\langle i\rangle} s_{26}^{\langle i\rangle}+s_{66}^{\langle i\rangle}} \sum_{i=1}^{n}\left\{\frac{a_{12}^{\langle i\rangle} s_{16}^{\langle i\rangle}+b_{12}^{\langle i\rangle} s_{26}^{\langle i\rangle}+s_{66}^{\langle i\rangle}}{h_{i}}\right\}
\end{aligned}
$$

